\title{
The collapse of a cavitation bubble in shear flows-A numerical study
}

\author{
Po-Wen Yu, Steven L. Ceccio, and Grétar Tryggvason \\ Department of Mechanical Engineering and Applied Mechanics, The University of Michigan, \\ Ann Arbor, Michigan 48109-2121
}

(Received 15 February 1995; accepted 30 June 1995)

\begin{abstract}
The collapse of a cavitation bubble is examined by direct numerical simulations of the NavierStokes equations, using a finite difference/front tracking technique. Bubbles in both a quiescent fluid as well as shear flows are examined. For quiescent fluid, the results are compared with theoretical and previous computational results. For bubbles in a shear flow it is shown that large shear can increase the rate of collapse, and for bubbles near boundaries shear can eliminate the re-entrant jet seen for bubbles in a quiescent flow. (c) 1995 American Institute of Physics.
\end{abstract}

\section{INTRODUCTION}

Cavitation bubbles which collapse near solid boundaries may cause surface erosion and harmful vibrations, as well as undesirable acoustic emission. Cavitation can occur in turbomachinery, on propulsors, and in hydraulic circuits. Consequently, considerable research has been conducted into the mechanism of cavitation bubble dynamics; see, for example, Naude and Ellis, ${ }^{1}$ Blake and Gibson, ${ }^{2,3}$ Shima and Nakajima, ${ }^{4}$ Lauterborn and Bolle, ${ }^{5}$ Shutler and Mesler, ${ }^{6}$ van der Meulen, ${ }^{7}$ Plesset and Chapman, ${ }^{8}$ and Mitchell and Hammitt. 9

Over the last few decades, numerical simulations of cavitating flows have improved our understanding of cavitation bubble dynamics. The first such computer simulations were done by Plesset and Chapman ${ }^{8,10}$ and Mitchell and Hammitt. ${ }^{9}$ Plesset and Chapman studied the collapse of an initially spherical cavitation bubble near a solid wall. The shape and velocity of the bubble interface were calculated for an axisymmetric bubble collapsing in an irrotational flow. Mitchell and Hammitt simulated the collapses of an axisymmetric bubble in an incompressible, viscous liquid. Both authors used a finite difference method based on the MAC method of Harlow and Welch." Later researchers have assumed that the cavitating liquid can be taken as inviscid, and the problem thus reformulated in terms of an integral equation over the bubble surface. See, for example, Blake and Gibson ${ }^{3,12}$ and Chahine. ${ }^{13-16}$ These boundary integral simulations have yielded much insight into cavitation bubble dynamics.

Cavitation bubbles often occur in complex vortical flows such as shear layers or tip vortices, and these flows have a strong influence on the growth and collapse of the bubble. Ceccio and Brennen, ${ }^{17}$ for example, observed that traveling cavitation bubbles forming in a flow near a solid surface will interact strongly with the boundary layer. The bubbles were stretched by the shear flow near the surface, and they often were observed to fission into several smaller bubbles before collapsing. Boundary integral simulations of cavitation bubbles generally do not allow any vorticity in the flow field, although Chahine has recently endeavored to include these interactions in his boundary integral simulations. ${ }^{18}$ Furthermore, cavitation may occur under such circumstances that the liquid viscosity influences the cavity dynamics. Considering a spherical bubble, Poritsky ${ }^{19}$ determined that changes in liquid viscosity can alter the pressure at the bubble surface and thus reduce the effective pressure differential between the fluid and the vapor. This will lead to a reduced rate of bubble growth and collapse. Shima and Tsujino ${ }^{20}$ observed that variation in viscosity caused by temperature gradients can damp the bubble collapse and in some cases eliminated the bubble rebound after the first collapse.

Three dimensional, direct numerical simulation of the dynamics of cavitation bubbles can reveal new insight into more complex cavitating flows such as flow with nonspherical, non-axisymmetric bubbles, and bubble clouds in vortical flows. In this work, the dynamics of cavitation bubbles is simulated directly, and the effects of fluid viscosity and surface tension are considered. The full NavierStokes equations are solved by a finite difference/front tracking method that allows a fully deformable bubble surface. Although our technique allows us to simulate the interaction of several bubbles with the flow field and nearby solid boundaries, we only report results from simulations of single cavitating bubbles here. A preliminary report of some of the work done here has been given at the 20th Naval Hydrodynamics Symposium in Santa Barbara on August 21-26, 1994.

\section{FORMULATION AND NUMERICAL APPROACH}

To simulate the dynamics of cavitation bubbles we use the Navier-Stokes equation in primitive variables. We consider flows where thermal effects are negligible and formulate a single vector equation for the unsteady viscous flow both inside and outside the bubble. In conservative form these are

$$
\begin{aligned}
\frac{\partial \rho \mathbf{u}}{\partial t}+\nabla \cdot(\rho \mathbf{u u})= & -\nabla p-\rho \mathbf{g}+\nabla \cdot(2 \mu \mathbf{D}) \\
& +\int \sigma \kappa \mathbf{n} \delta\left(\mathbf{x}-\mathbf{x}_{f}\right) d a .
\end{aligned}
$$

Here, $\mathbf{D}$ is the deformation tensor, $\mathbf{u}$ is the velocity vector, $p$ is the pressure, and $\rho$ and $\mu$ are the discontinuous density and viscosity fields, respectively. Here $\sigma$ is the surface tension, $\kappa$ is twice the mean curvature and $\mathbf{n}$ is a normal to the bubble boundary. Here $\mathrm{g}$ is the gravity acceleration. Notice that surface tension forces have been added as a delta function, only affecting the equations where the interface exists. 
The above equations must be supplemented by additional conditions leading to an equation for the pressure field. Here, we assume that the ambient fluid is incompressible, so that

$$
\boldsymbol{\nabla} \cdot \mathbf{u}=0
$$

which, when combined with the momentum equations leads to a non-separable elliptic equation for the pressure. For the cavitating bubble we assume that the pressure inside the bubble is uniform and equal to the fluid vapor pressure $p_{v}$. Additional pressure boundary conditions are assigned along one or more sides of the computational domain. We also take the density and viscosity of the ambient fluid to be constant. Inside and outside the bubble, the viscosity and density remain constant, but there are discontinuities in these properties at the bubble boundary.

The process of bubble collapse is influenced by the initial size of the bubble, the pressure difference between the vapor and the liquid far away from the bubble surface, a characteristic shear rate of the flow surrounding the bubble, and the physical properties of the liquid. Thus, we form the following three non-dimensional parameters:

$$
\begin{aligned}
& \alpha=\omega r_{0} \sqrt{\frac{\rho}{\Delta p}}, \\
& \beta=\frac{\mu}{r_{0}} \sqrt{\frac{1}{\rho \Delta p}}, \\
& \gamma=\frac{\sigma}{r_{0} \Delta p} .
\end{aligned}
$$

Here $\omega$ is a characteristic shear rate and $r_{0}$ is a bubble length scale, typically the bubble initial radius. The density and viscosity are those of the liquid. Here $\Delta p$ is the difference between the far field pressure and the vapor pressure of the fluid. A velocity scale can be formulated with the pressure difference and fluid density:

$$
U_{c}=\sqrt{\frac{\Delta p}{\rho}}
$$

$U_{c}$ scales the collapse velocity of the bubble. Thus, the parameter $\alpha$ can be interpreted as a velocity ratio:

$$
\alpha=\frac{U_{s}}{U_{c}},
$$

where $U_{s}=\omega r_{0}$ is a velocity scale based on the characteristic shear rate. The viscosity parameter $\beta$ is a modified Reynolds number:

$$
\beta=\frac{\mu}{\rho r_{0} U_{c}}=\frac{1}{\mathbf{R e}_{\beta}} .
$$

The surface tension parameter $\gamma$ is a modified Weber number:

$$
\gamma=\frac{\sigma}{U_{c}^{2} \rho r_{0}}=\frac{1}{\mathbf{W e}_{\gamma}} .
$$

The same variables can also be used to generate a time scale, the Rayleigh time:

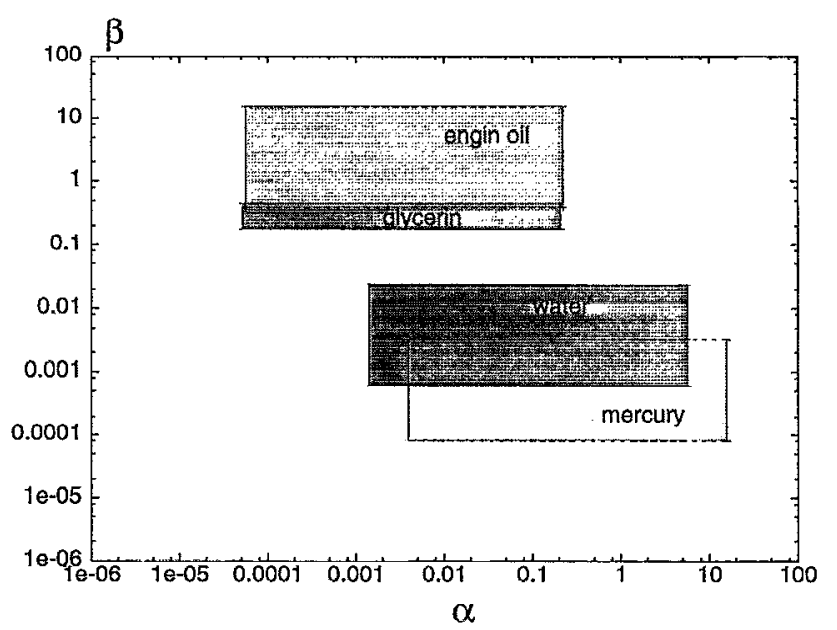

FIG. 1. Ranges of $\alpha$ and $\beta$ for bubble cavitation in a typical wall bounded shear flow with flow conditions defined in the text. Here $\alpha=\omega r_{0} \sqrt{\rho / \Delta p}$, $\beta=\mu / r_{0} \sqrt{\rho \Delta p}$.

$$
t_{R}=r_{0} \sqrt{\frac{\rho}{\Delta p}} .
$$

The Rayleigh time is proportional to the collapse time of a spherical vapor bubble in an unbounded fluid, and it has been used to non-dimensionalize the time scale of our results. For an initially spherical bubble near a wall, a non-dimensional position parameter, $\delta^{*}$, is defined as

$$
\delta^{*}=\frac{d}{r_{0}},
$$

where $d$ is the distance from the center of the bubble to the wall. In the following calculations, we do not consider the flow of the vapor within the cavitation bubblc. This approximation is often valid up until the final stages of the bubble collapse.

In order to evaluate the non-dimensional parameters $(\alpha, \beta, \gamma)$ for typical cavitating flows, the following estimates may be used. The cavitation number, $\sigma_{c}$, is defined as

$$
\sigma_{c}=\frac{P-P_{u}}{\frac{1}{2} \rho U^{2}},
$$

where $P$ is the free stream pressure, $P_{v}$ is the liquid vapor pressure, and $1 / 2 \rho U^{2}$ is the dynamic head of the flow.

For cloud cavitation, the bubble size is often on the order of $100 \mu \mathrm{m}$, and the maximum cavitation number, $\sigma_{c}$, in the region of the collapsing cloud is often on the order of unity, making the pressure difference approximately equal to the dynamic head of the flow, $\frac{1}{2} \rho U^{2}$. For $20^{\circ} \mathrm{C}$ water flowing at $10 \mathrm{~m} / \mathrm{s}$, this translates to $\Delta p$ of approximately $50 \mathrm{kPa}$. Small cavitation bubbles often occur within the boundary layer near a solid boundary. ${ }^{21,22}$ If the boundary layer thickness is approximately ten times the bubble diameter or $1 \mathrm{~mm}$, the non-dimensional parameters are $\alpha=0.14, \beta=0.0012$, and $\gamma=0.014$. Of course, these estimates are more appropriate to dilute bubble clouds, where the bubbles do not interact strongly. Figure 1 shows the parameter ranges for different fluids when $r_{0}$ varies from 50 to $200 \mu \mathrm{m}$, and the dynamic 
head is calculated for flows with velocities ranging from 1 to $10 \mathrm{~m} / \mathrm{s}$, and the boundary layer thickness $\delta$ is equal to $\sqrt{\mu / \rho U}$.

To solve the Navier-Stokes equation we use a fixed, regular, staggered grid and discretize the momentum equations using a conservative, second order centered difference scheme for the spatial variables and an explicit first order time integration method. The pressure equation, which is non-separable due to the difference in density between the bubbles and the ambient fluid, is solved by a Black and Red SOR scheme. The novelty of the scheme is the way in which the boundary, or the front, between the vapor and the ambient fluid is tracked. The front is represented by separate computational points that are moved by interpolating their velocity from the grid. These points are connected hy triangular elements to form a front that is used to keep the density and viscosity stratification sharp and to calculate surface tension forces. At each time step information must be passed between the front and the stationary grid. This is done by a method similar to the one discussed by Unverdi and Tryggvason, ${ }^{23}$ that spreads the density jump to the grid points next to the front and generates a smooth density field that changes from one density to the other over two to three grid spaces. While this replaces the sharp interface by a smoother grid interface, all numerical diffusion is eliminated since the grid-field is reconstructed at each step. The surface tension forces are computed from the geometry of the interface and distributed to the grid in the same manner as the density jump. As the bubbles move and deform, it is necessary to add and delete points at the front and to modify the connectivity of the points, to keep the front elements of approximately equal size and as "well shaped" as possible. The details are described in Unverdi \& Tryggvason. ${ }^{23}$ This method has been used to simulate a number of non-cavitating multi-fluid problems such as the head-on collisions of drops, ${ }^{24,25}$ the effect of contaminants on the rise of buoyant bubbles, ${ }^{26}$ the coalescence of drops of different sizes, ${ }^{27}$ and the lift of deformable bubbles rising in a shear flow. ${ }^{28}$ Typical time for the simulations presented here are about 2-3 days on a IIP715 workstation for a $32^{3}$ grid.

\section{RESULTS AND DISCUSSIONS}

\section{A. A bubble in a quiescent fluid}

In order to validate our implementation of the numerical method and to assess how rapidly it converges under grid refinement, we have computed the collapse of a spherical vapor bubble in an unbounded fluid and compared the results with the predictions of the Rayleigh-Plesset equation. The Rayleigh-Plesset ${ }^{29}$ equation describes the dynamics of a spherical bubble with radius $R(t)$ in an infinite fluid including the effects of liquid viscosity and surface tension.

The equation can be rewritten in dimensionless form as follows:

$$
\bar{R} \frac{d^{2} \bar{R}}{d t^{2}}+\frac{3}{2}\left(\frac{d \bar{R}}{d t}\right)^{2}+\frac{4 \beta}{\bar{R}} \frac{d \bar{R}}{d t}+\frac{2 \gamma}{\bar{R}}+1=0 .
$$

Here, dimensionless radius $\check{R}$ is defined as $R / r_{0}$.

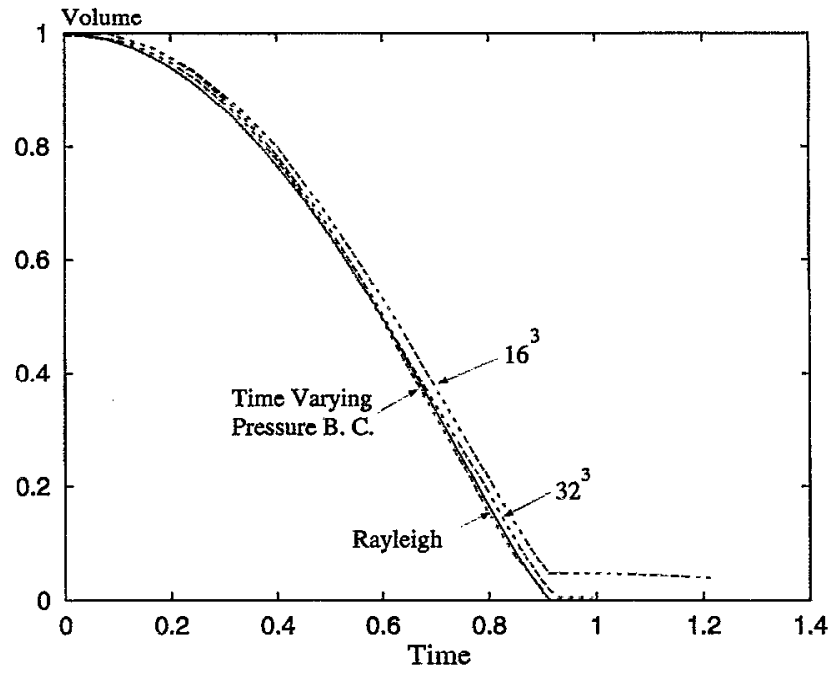

FIG. 2. The collapse of an unbounded spherical bubble computed on a $16^{3}$ and a $32^{3}$ grid. The volume of the bubble is plotted versus dimensionless time. Also plotted is the volume predicted by the Rayleigh-Plesset equation and the volume calculated with a time varying pressure boundary.

First, a comparison is made between the solution of the inviscid Rayleigh-Plesset equation and computations using the current method. The computations are done with a fully three-dimensional method, but in a relatively small computational domain. The pressure is prescribed on the boundary of the computational domain (whose sides are twice the diameter of the initial bubble) instead of at infinity as is the case for the collapse of a bubble in an unbounded fluid. We have checked the effect of this approximation in two ways. First, we have simulated the collapse in computational domain twice the original size, and second, we have specified the exact time varying pressure, as computed from the Rayleigh-Plesset equation, on the boundaries of the computational domain. In both cases the deviations from the original case are small. Figure 2 shows the radius of the bubbles versus dimensionless time for both a $16^{3}$ and a $32^{3}$ grid as well as the theoretical result. The results show that the evolution is well predicted during the early stages by both resolutions, but a loss of resolution occurs when the bubble shrinks, eventually leading to a sudden deviation from the analytical prediction for both cases. For the coarser grid, this takes place when the bubble volume is about $5 \%$ of the original volume. At this point the bubble is resolved by only two and one half meshes. For the finer grid, comparible resolution also leads to a departure from the analytical solution, but now the bubble volume is $1 \%$ of the original volume. In the same figure, the result for the time varying pressure boundary condition is also plotted. These results, as expected, show better agreement with the exact solution than the constant boundary pressure cases.

Next, the collapse of a spherical bubble in an unbounded viscous fluid is examined for several different viscosity and surface tension parameters ( $\beta$ and $\gamma$ ). The results are compared with those obtained by the Rayleigh-Plesset equation. Figure 3 shows the volume of the bubbles versus dimension- 


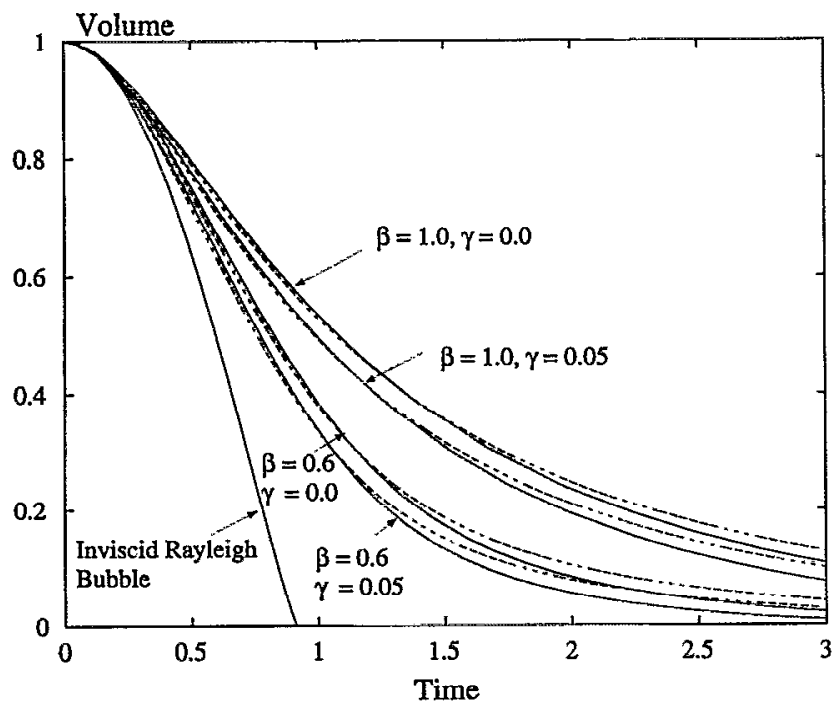

FIG. 3. The collapse of an unbounded spherical bubble in an initially quiescent fluid computed on a $32^{3}$ grid for $\gamma=0, \beta=1.0$ and $0.6, \gamma=0.05$, $\beta=1.0$ and 0.6 . The volume of the bubble is plotted versus dimensionless time (dashed lines). Also plotted are the volumes as predicted by both viscous and inviscid Rayleigh-Plesset equations (solid lines).

less time. All computations were done on a $32^{3}$ grid with time varying pressure boundary conditions. These results agree well with solutions of Rayleigh-Plesset equation until resolution is lost within the bubble. As noted by Poritsky, ${ }^{19}$ an increase in $\beta$ results in a reduced bubble collapsing rate, and an increase in $\gamma$ results in an acceleration of the bubble collapse. Comparison between solutions of the RayleighPlesset equation and the current method have also been performed for the cases of two-dimensional (cylindrical) and one-dimensional (a plane liquid/vapor interface) bubbles. In both these cases, the current method compared well with the analytical solution until resolution was lost within the bubble.

Cavitation bubbles collapsing near a solid boundary in an initially motionless fluid will not collapse uniformly. Instead, a jet of liquid will form as the surface of the bubble farthest away from the solid boundary collapses faster than the surface closest to the wall. The formation of this "reentrant jet" is a well known phenomena and has been examined both numerically ${ }^{1,3}$ and experimentally. ${ }^{1,5,30}$ Figure 4 shows the evolution of an initially spherical bubble collapsing near a wall $\left(\delta^{*}=1.5\right)$ in an initially quiescent fluid. The bubble is shown at four unequal time intervals, and the view is from the side and above revealing the three dimensional aspects of the evolution. The computational domain is a cube whose bottom is shown in the figure. The domain is resolved by a $32^{3}$ grid and the initial bubble diameter is 0.5 times the side length of the computational domain. The pressure is specified at the top of the computational domain, and both horizontal boundaries are periodic. For this case $\alpha=0$, $\beta=0.007$ and $\gamma=0.288$. As time proceeds, the formation of a jet is clearly evident.

Figurc 5 shows the normalized volume versus dimensionless time for three different spatial resolutions. The rate of volume reduction converges well initially. However, the
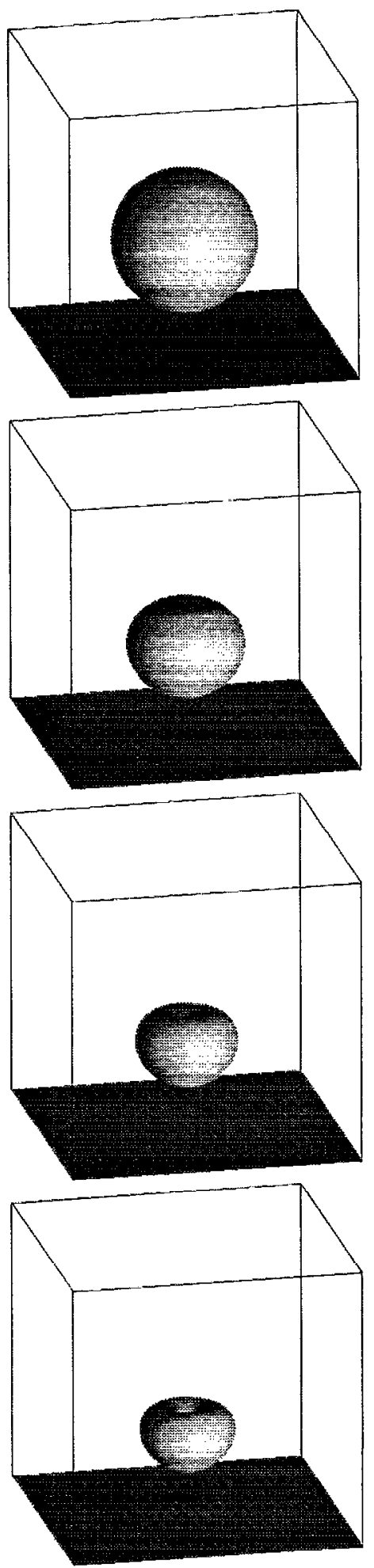

FIG. 4. Computations of the collapse of a cavitation bubble in an initially quiescent fluid near a wall. The computations are done in a cubc computational box resolved by a $32^{3}$ grid. The initial bubble diameter is 0.5 times the side length of the box. Here $\alpha=0, \beta=0.007, \gamma=0.288$, and $\delta^{*}=1.5$. The bubble is shown at times $0.0,0.94,1.1$, and 1.16 . The visualization of the bubble was done by AVS.

collapse rate is reduced for the lower resolution cases during the later stages of the collapse. This results from a loss of resolution in the liquid jet, which is wider for the lower 


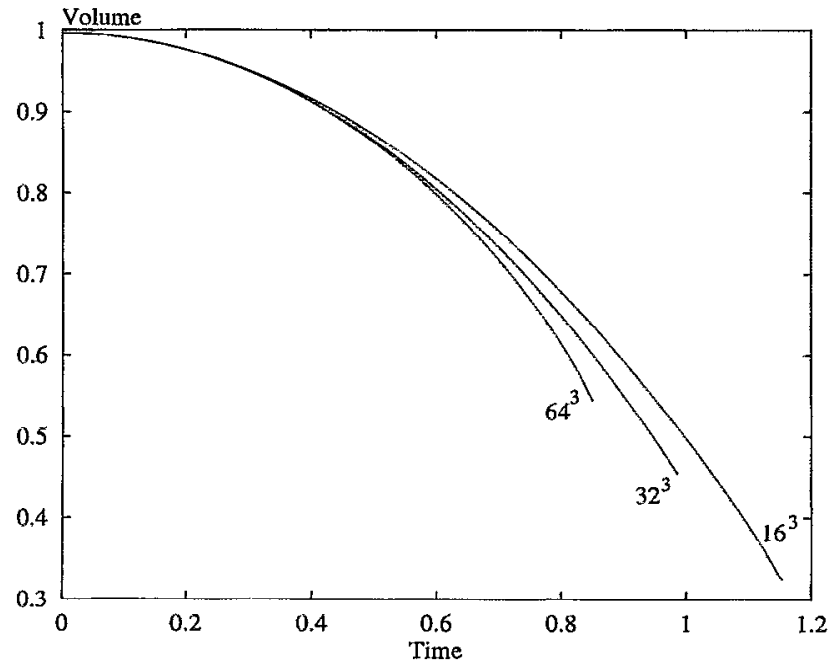

FIG. 5. The normalized volume of the bubble in Fig. 4 versus dimensionless time. Also plotted are the results from calculations on both a coarse $16^{3}$ grid as well as a finer $64^{3}$ grid. The nommalized volume is defined as the ratio of bubble volume over initial bubble volume.

resolution runs. As a result, the collapse is slowed. This effect is similar to that observed during the last stages of the purely spherical collapse. Figure 6 shows the maximum downward velocity of the bubble surface versus dimensionless time for three different resolutions. The jet velocity increases dramatically as a jet forms. Also included in the figure are the numerical results of Plesset and Chapman. ${ }^{8}$ The grid (in cylindrical coordinates) used by Plesset and Chapman for the axisymmetric simulation sets the radius of outer boundary equal to 40 meshes and the radius of the initially spherical bubble equal to 5 meshes. The difference in time when the jet accelerates between the present simulation and that of Plesset and Chapman suggests that the jet speed is not

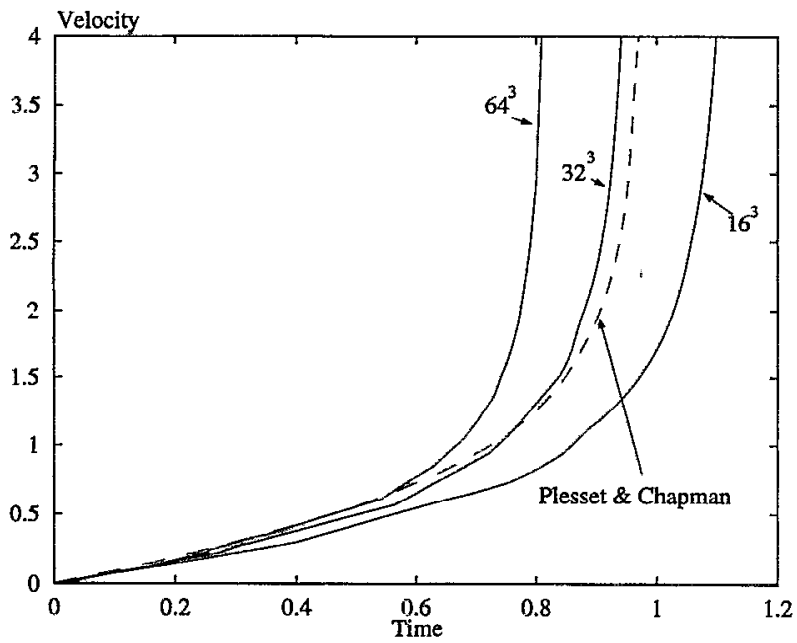

FIG. 6. The maximum downward velocity of the bubble surface versus dimensionless time for the collapse of an initially spherical bubble near a solid boundary in an initially quiescent fluid. Here $\delta^{*}=1.5, \beta=0$. Results for three resolutions are shown along with the results from Plesset and Chapman. The velocity is non-dimensionalized by $\sqrt{\Delta p / \rho}$.

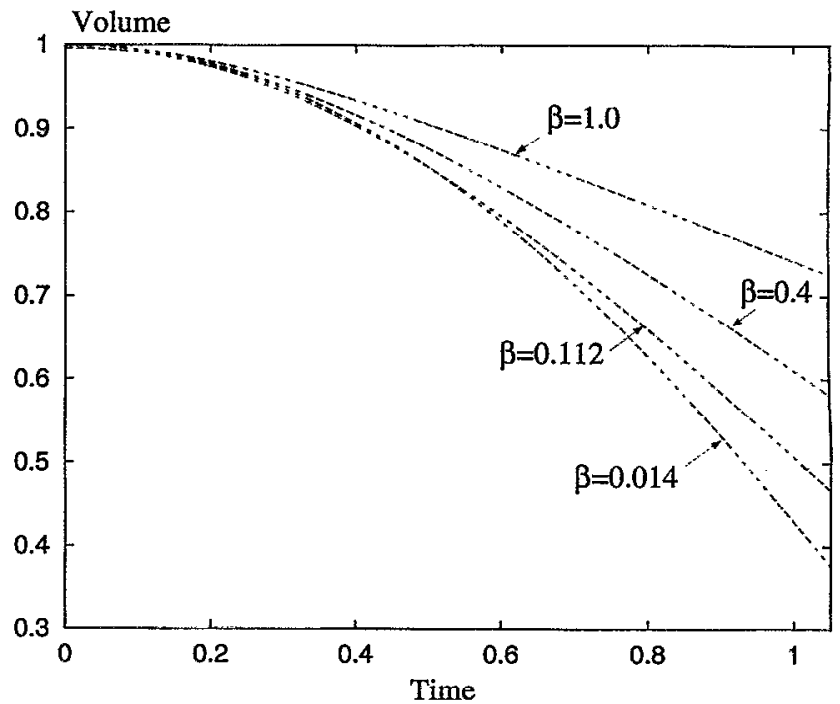

FIG. 7. The normalized volume versus dimensionless time for an initially spherical bubble collapsing near a wall in an initially quiescent fluid. Here $\beta=0.014,0.112,0.4$ and 1.0 respectively. The computations are done in a cube computational domain resolved by a $32^{3}$ grid.

fully resolved in neither the present computations nor theirs, although experiments support the general validity of both simulations. ${ }^{5}$

Liquid viscosity can reduce the rates of bubble growth and collapse compared to those predicted by inviscid theory. Figure 7 shows the normalized bubble volume versus dimensionless time for four different non-dimensional viscosity parameters, $\beta$. For these cases, $\alpha=0$ and $\delta^{*}=1.5$. As $\beta$ is increased, the collapse rate is reduced, the evolution of the bubble shape is modified, and the jet formation is delayed. These trends are similar to those noted by Poritsky ${ }^{19}$ for the collapse of a spherical bubble in an unbounded viscous fluid. Variation in surface tension may also influence the collapse

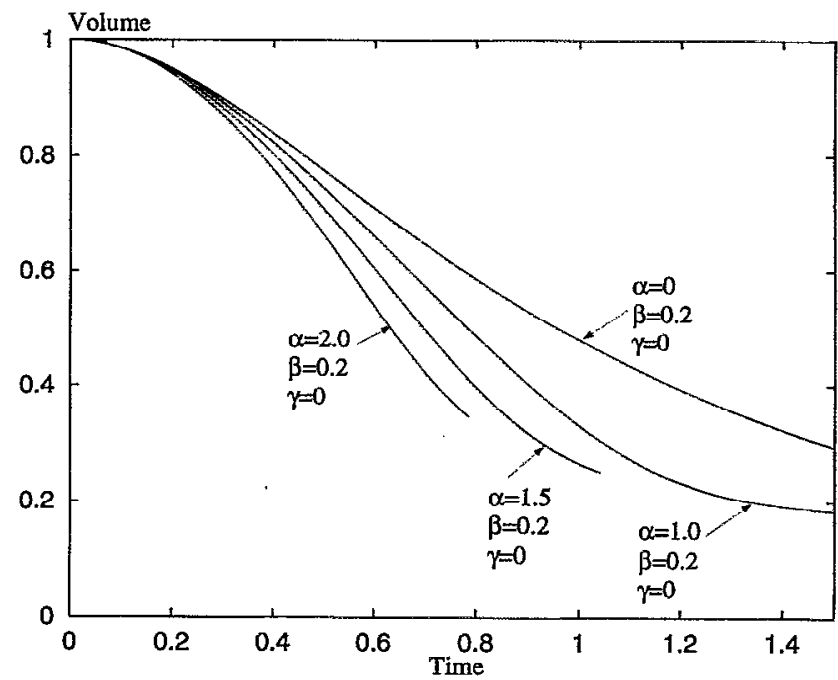

FIG. 8. The normalized volume versus dimensionless time for an initially spherical bubble collapsing in a shear flow. Here $\alpha=1.0,1.5$ and $2.0, \beta=$ 0.2 and $\gamma=0$. The computations are done in a cube computational domain resolved by a $32^{3}$ grid. 

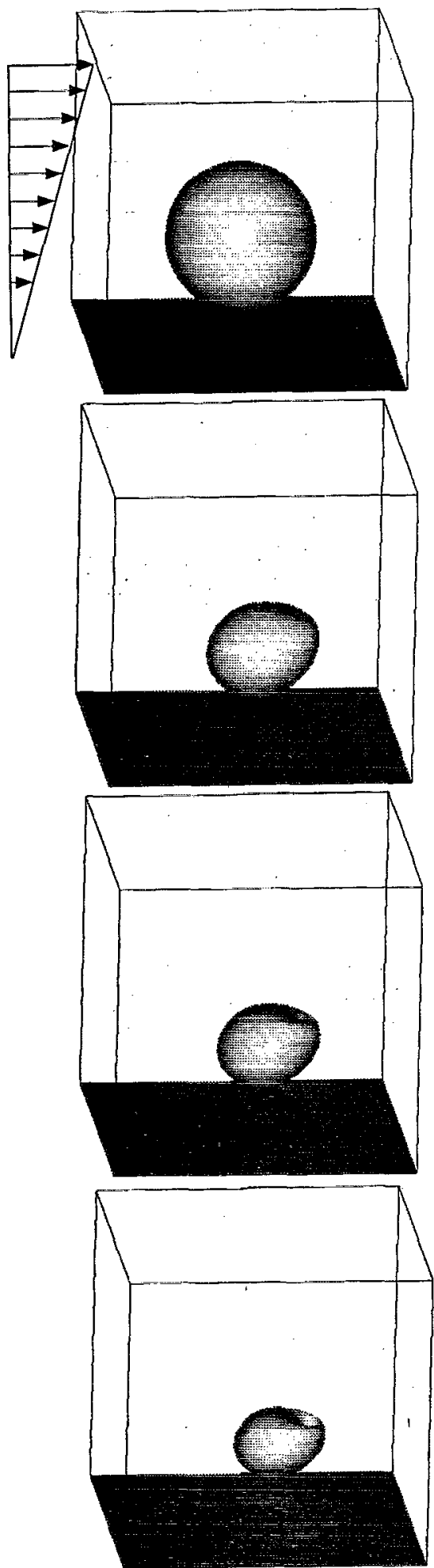

FIG. 9. Computations for the collapse of an initially spherical cavitation bubble in a shear flow near a solid boundary. The computations are done in a cube computational box resolved by a $32^{3}$ grid. The initial bubble diameter is 0.5 times the side length of the box. Dinensionless parameters are $\alpha=0.15, \beta=0.022, \gamma=0.288$ and $\delta^{*}=1.5$. The bubble is shown at dimensionless time $0.0,0.94,1.1$ and 1.16 from top to bottom.

of a cavitation bubble ncar a wall. It is expected that an increase in surface tension will increase the initial collapse rate. Shima and Najajima ${ }^{4}$ investigated the collapse of a non-

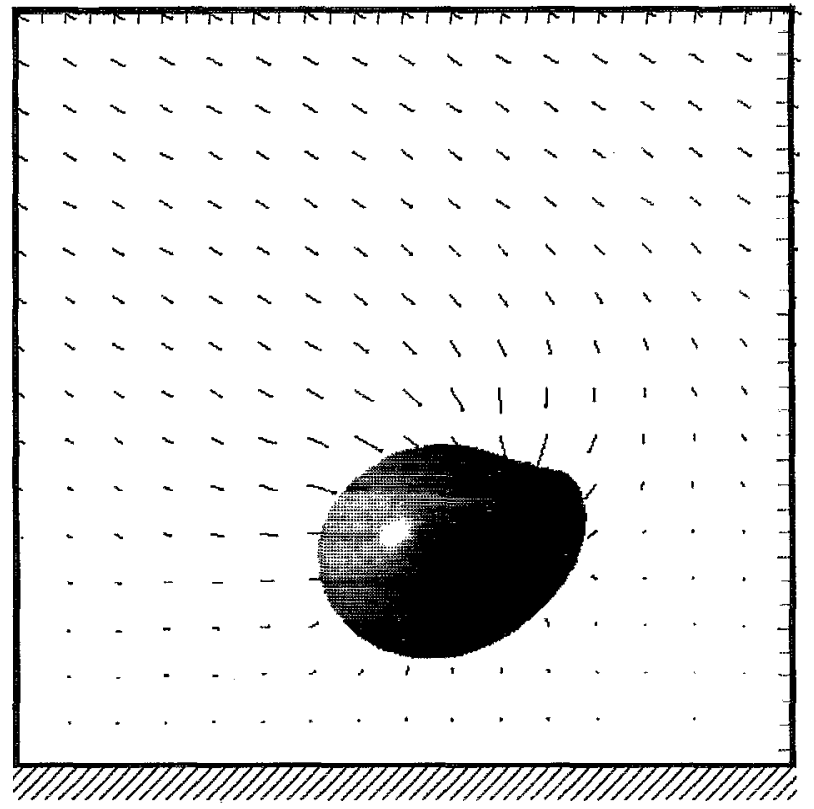

FIG. 10. The velocity field in the center plane of a bubble collapsing near a solid boundary in a shear flow. The vectors shown here were plotted at every other grid point. The conditions are the same as shown in Fig. 9.

hemispherical (prolate hemispheroid) bubble attached to a solid wall and determined that an increase in surface tension increased the collapse speed during the final stages of collapse. In the current simulations, the influence of increasing surface tension during the later stages of collapse was offset by the loss of resolution. Thus, this phenomenon was not investigated here.

\section{B. A bubble in a shear flow}

Cavitation bubbles often grow and collapse within a vortical flow. The direct numerical simulation of cavitation bubbles presented here permits the simulation of cavitation bubbles in a flow with vorticity. First, the collapse of a cavitation bubble in a shear flow is examined. An initially spherical bubble is placed in a shear flow which is characterized by the shear parameter $\alpha$. The boundary condition is set to be periodic in the shear flow direction. Figure 8 shows the normalized bubble volume versus dimensionless time for $\alpha=$ $0.0,1.0,1.5$ and 2.0. As the shear rate is increased, the collapse rate also increases. For high shear collapse, the rate approaches that of an inviscid bubble without shear. Note that the pressure boundary condition is not symmetric for the sheared case, and thus the computed solution for the case $\alpha=0, \beta=0.2$ does not match the exact solution as closely as the previous unsheared bubble computation. The pressure boundary condition used could be made time varying to provide a closer match to the exact solution.

The effect of both shear and the presence of a solid boundary is next examined. Figure 9 shows the collapse of a bubble in a shear flow where $\alpha=0.15, \beta=0.022$, $\gamma=0.288$ and $\delta^{*}=1.5$. A linear velocity distribution is imposed initially. The bubble is shown at four dimensionless times (with unequal time intervals) as in Fig. 4. Figure 10 shows the velocity field in the center plane at dimensionless 

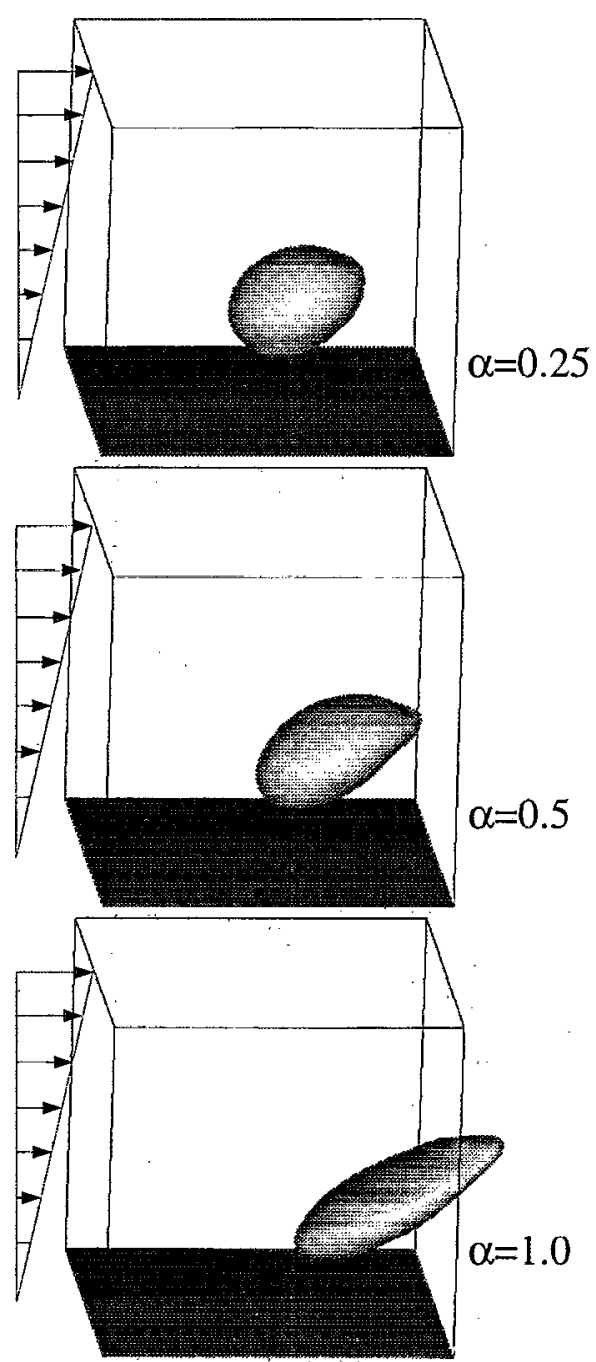

FIG. 11. The collapse of three bubbles in a shear flow near a wall. The initial velocity profile is shown. The computations are done in a cube computational box resolved by a $32^{3}$ grid. The initial bubble diameter is 0.5 times the side length of the box. The bubbles are all shown at $t=1.04$, and $\beta=0.0222$ and $\gamma=0.288$ in all cases. Here $\alpha=0.25,0.5$ and 1.0 , respectively, from top to bottom.

time $t=1.12$. The velocity vector is plotted at every other grid point and shows clearly the asymmetry in the field. The location of the jet, which was at the center for the case without shear, is now moved toward the downstream direction, and the bubble is stretched into a slightly asymmetric "saucer" shape (see the bottom bubble in Fig. 9).

Three simulations with stronger shear rates are shown in Fig. 11. The three bubbles in Fig. 11 are plotted at the same dimensionless time with shear rate increasing from top to bottom. For the sheared cases the jet is smaller when compared to the non-shear case, and the jet location is moved farther downstream as the shear increases until jet formation is not observed anymore. Thus, the presence of shear can lead to dramatic changes in the bubble shape and prevent the formation of the re-entrant jet.

The normalized volume of the sheared bubbles is plotted as a function of time in Fig. 12 versus time for four different

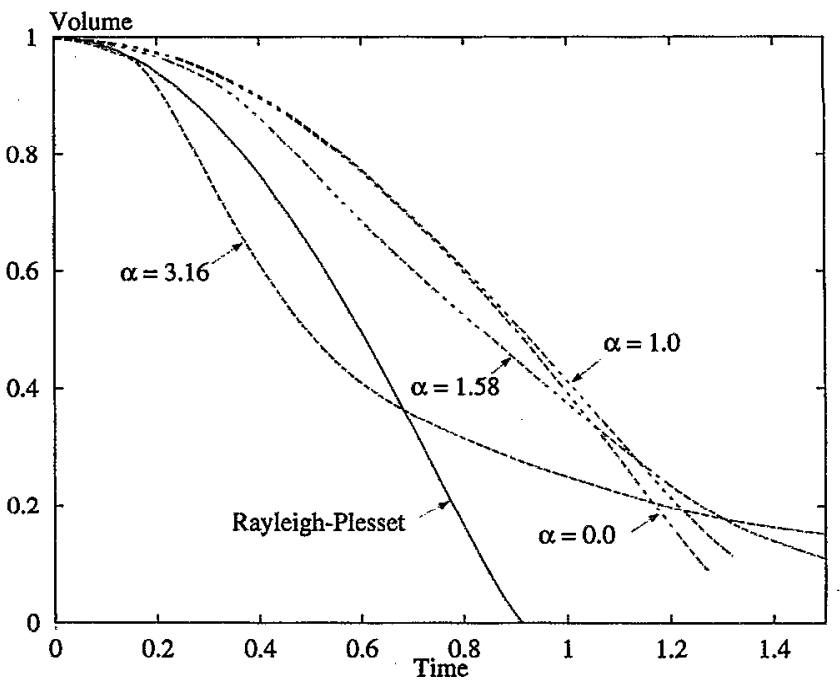

FIG. 12. The normalized volume versus dimensionless time plot. The collapse of an initially spherical bubble near a wall and in a shearing flow. Here $\alpha=0.0,1.0,1.58$ and 3.16. $\beta=0.022, \gamma=0.288, \delta^{*}=1.5$ for all cases (dashed lines). Also plotted are the results predicted by the Rayleigh-Plesset equation for the collapse of a spherical bubble in an unhounded fluid (solid line).

shear rates. For the first two cases with shear rates equal to 0.0 and 1.0 the collapse rates are almost identical. The shearing does not significantly effect the collapse rate of the bubble as the time scale associated with the bubble collapse is shorter than that associated with the shearing of the fluid. In these cases, a re-entrant jet may form. For more highly sheared cases, however, the stretching increases the surface area even more. The jet does not form, and the bubble collapsc rate increases sharply. Once the stretched bubble reaches the resolution limit, the collapse rate slows down. Notice that for the highest shear rate in Fig. 12, the collapse rate is even larger than the theoretical rate of collapse for a bubble in an infinite fluid.

Traveling bubble cavitation can occur when small gas bubbles (cavitation nuclei) are convected into a region of low pressure. The nuclei will grow into macroscopic cavitation bubbles, and these cavitation bubbles can interact strongly with the shear flow near a solid boundary (Ceccio and Brennen ${ }^{17}$ ). The computational method described here can be used to examine the growth and collapse of travelling cavitation bubbles, and a simplified model is presented for the purpose of illustration. Figure 13 shows the interaction of a cavitating bubble with a thin boundary layer as the bubble moves near a solid surface. For large traveling cavitation bubbles which convect over a solid boundary, a Lagrangian model is employed. The flow is modeled by placing a small cavitation bubble close to a solid boundary (with $\delta^{*}=1$ ). The wall is initially stationary, and the pressure inside the bubble is set to be larger than the surrounding quiescent fluid, resulting in bubble growth. The bubble expands and takes on a semi-spherical shape as it impinges on the solid wall (see the first bubble in Fig. 13). The far field pressure is then increased to initiate bubble collapse, and the bottom boundary is impulsively set into motion. As the boundary layer grows in thickness the semi-spherical bubble begins to 


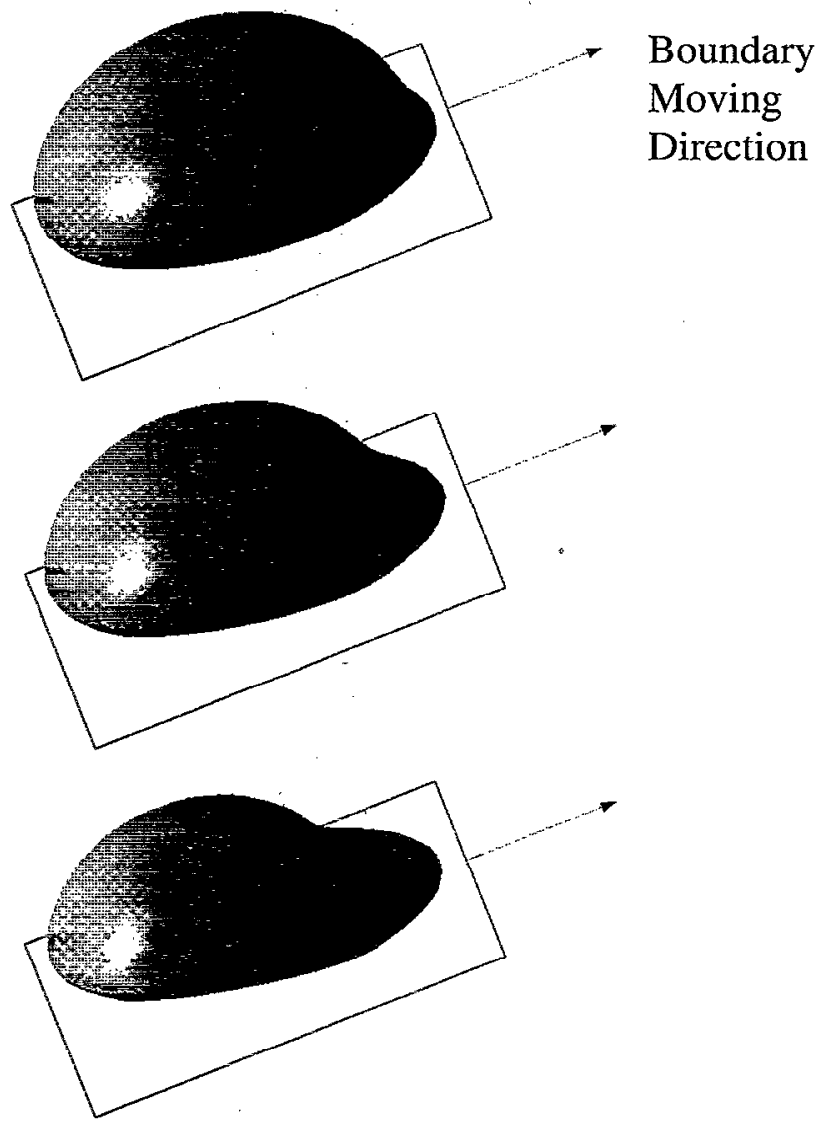

FIG. 13. The smearing effect result from the interaction between a cavitation bubble and a moving boundary. The bubble is deformed by the shear flow induced by the moving boundary.

interact with the shear flow, and the shape of the lower half of the bubble is smeared out. This effect is observed in the lower two bubble shapes shown in Fig. 13. The bubble/shear layer interactions presented here are qualitatively similar to those observed experimentally for travelling cavitation bubbles moving near a solid surface (see Jean-Piere Le Goff ${ }^{31}$ and Ivany ${ }^{32}$ ). In this simulation, the "(rail" of vapor left behind the bubble is thicker than those observed experimentally due to the limited resolution of the calculation.

\section{CONCLUSIONS}

In this paper, the dynamics of cavitating bubbles is explored using direct numerical simulations. The full NavierStokes equations are solved by a finite different/front tracking method that allows a fully deformable interface between the bubbles and the ambient fluid. The results for the simulation of a spherical bubble collapsing in a quiescent unbounded fluid were compared to the numerical solution of the Rayleigh-Plesset equation. The solution using the front tracking method converges to the Rayleigh-Plesset solution with increasing spatial resolution. Furthermore, similar validation have been performed for two-dimensional (cylindrical bubble) and one-dimensional (line-interface) bubble collapse. ${ }^{33}$ For all these cases, reasonable accuracy can be achieved with a moderate resolution. The case of a threedimensional bubble collapsing near a solid boundary was computed and compared to the solution of Plesset and Chapman, and the solutions were found to be similar. Both solutions predict the formation of a re-entrant jet.

The influence of shear, viscosity, and surface tension on the collapse of a single cavitation bubble were examined. It was found that for sufficiently strong shear $(\alpha>1)$ the collapse rate of the bubble will be significantly increased, and the formation of the re-entrant liquid jet will be eliminated. These effects are due to the elongation of the bubble under the influence of the shearing flow. Increasing viscosity led to a reduction in the rate of bubble collapse and increasing surface tension accelerates the collapse. The front tracking method was also used to simulate the distortion of a traveling cavitation bubble.

\section{ACKNOWLEDGMENT}

This work is supported by the Office of Naval Research under Contract Nos. N00014-92-1063 and N00014-91-J1084 , with Dr. E. P. Rood as technical monitor.

IC. F. Naude and A. T. Ellis. "On the mechanism of cavitation damage by non-hemispherical cavities in contact with a solid boundary," Trans. ASME J. Basic Eng. 83, 648 (1961).

${ }^{2}$ J. R. Blake and D. C. Gibson, "Growth and collapse of a vapor cavity near a free surface," J. Fluid Mech. 111, 123 (1981).

${ }^{3}$ J. R. Blake, B. B. Taib, and G. Doherty, "Transient cavities near boundaries. Part I. Rigid boundary," J. Fluid Mech. 170, 479 (1986). .

${ }^{4}$ A. Shima and K. Nakajima, "The collapse of a non-hemispherical bubble attached to solid wall," J. Fluid Mech. 80, 369 (1977).

${ }^{5} \mathrm{~W}$. Lauterborn and $\mathrm{H}$. Bolle, "Experimental investigations of cavitation bubble collapse in the neighborhood of a solid boundary," J. Fluid Mech. 72, 391 (1975).

${ }^{6}$ N. D. Shutler and R. B. Mesler, "A photographic study of the dynamics and damage capabilities of bubbles collapsing near solid boundaries," Trans. ASME J. Basic Eng. 87, 511 (1965).

${ }^{7}$ J. H. J. van der Meulen, "A study of the collapse of laser-induced bubbles in a flow near a boundary," in ASME Cavitation and Multiphase Flow (American Society of Mechanical Engineers, New York, 1987), pp. 21-25.

${ }^{8}$ M. S. Plesset and R. B. Chapman, "Collapse of an initially spherical vapor cavity in the neighborhood of a solid boundary," J. Fluid Mech. 47, 238 (1971).

${ }^{9}$ T. M. Mitchell and F. H. Hammitt. "Asymmetric cavitation bubble collapse," Trans. ASME J. Fluid Eng. 95, 29 (1973).

${ }^{10}$ R. B. Chapman and M. S. Plesset, "Nonlinear effects in the collapse of a nearly spherical cavity in a liquid," Trans. ASME, J. Basic Eng. 94, 142 (1972).

${ }^{11} \mathrm{~F} . \mathrm{H}$. Harlow and J. E. Welch, "Numerical calculation of time-dependent viscous incompressible flow of fluid with free surface," Phys. Fluids. 8 , 2182 (1965).

${ }^{12}$ J. R. Blake, B. B. Taib, and G. Doherty, "Transient cavities near boundaries. Part I. Free Surface," J. Fluid Mech. 170, 479 (1986).

${ }^{13} \mathrm{G}$. L. Chahine, "Numerical modeling of the dynamic behavior of bubbles in nonuniform flow," Num. Methods Multiphase Flows 91, 57 (1990).

${ }^{14} \mathrm{G}$. L. Chahine and R. Duraiswami, "Dynamical interactions in a multibubble cloud," Trans. ASME J. Fluid Eng. 114, 680 (1992).

${ }^{15} \mathrm{G}$. L. Chahine, "Strong bubble/bubble and bubble/flow interactions," Bubble Dynamics and Interface Phenomena (Kluwer Academic, Dordrecht, 1994), pp. 181-195.

${ }^{16} \mathrm{~S}$. Green, "Fluid vortices," Bubble Interactions with Vortices, edited by G. L. Chahine (Kluwer, New York, 1995), Chap. XVIII, pp. 783-825.

${ }^{17}$ S. L. Ceccio and C. E. Brennen, "Observation of the dynamics and acoustics of travelling bubble cavitation," J. Fluid Mech. 233, 663 (1991).

${ }^{18} \mathrm{G}$. L. Chahine, "Bubble dynamics and cavitation inception in non-uniform flow fields," in Proceedings of The 20th Symposium on Naval Hydrodynamics (National Academy Press, Washington, DC, 1994), pp. 42-61.

${ }^{19} \mathrm{H}$. Poritsky, "The collapse or growth of a spherical bubble or cavity in a 
viscous fiuid," in Proceedings of the Ist National Congress on Applied Mechanics (ASME, New York, 1952), pp. 823-825.

${ }^{20} \mathrm{~A}$. Shima and T. Tsujino, "The behavior of bubbles in non-Newtonian lubricants," Trans. ASME J. Lub. Technol. 99, 455 (1977).

${ }^{21}$ M. Maeda, II. Yamaguchi, and H. Kato, "Laser holography mcasurement of bubble population in cavitation cloud on a foil section," in ASME Cavitation and Multiphase Flow Forum (American Society of Mechanical Engineers, New York, 1991), pp. 67-75.

${ }^{22} \mathrm{H}$. Yamaguchi, H. Kato, A. Kamijo, and M. Maeda, "Development of a laser holography system for the measurement of cavitation bubble clusters," in ASME Cavitation and Multiphase Flow Forum (American Society of Mechanical Engineers, New York, 1990), p. 115.

${ }^{23}$ S. O. Unverdi and G. Tryggvason, "Computations of multi-fluid flows," Physica D 60, 70 (1992).

${ }^{24}$ M. R. Nobari, Y.-J. Jan, and G. Tryggvason, "Head-on collisions of drops- a numerical investigation," submitted to Phys. Fluids.

${ }^{25} \mathrm{M}$. R. Nobari and G Tryggvason, "Numerical simulations of drop collisions," in Proceedings of the AIAA Meeting and Exhibit (AIAA, Washington, DC, 1993).
${ }^{26}$ Y.-J. Jan and G. Tryggvason, “A computational study of contaminated bubbles at finite Reynolds number," submitted to Phys. Fluids.

${ }^{27} \mathrm{M}$. R. Nobari and G Tryggvason, "Numerical simulation of three dimensional drop collisions," submitted to J. Fluid Mech.

${ }^{28}$ E. Ervin, "Computations of bubbles and drops in a shear flow," Ph.D. thesis, University of Michigan, 1993.

${ }^{29}$ M. S. Plesset, "The dynamics of cavitation bubbles," Trans. ASME. J. Appl. Mech. 71, 277 (1949).

${ }^{30} \mathrm{C}$. L. Kling and F. G. Hammitt, "A photographic study of spark-induced cavitation bubble," Trans. ASME J. Basic Eng. 94, 825 (1972).

31 J.-P. Le Goff, "Nuclei and cavitation," 14th Symposium on Naval Hydrodynamics (National Academy Press, Washington, DC, 1983), pp. 215242; p. 232, photo 1 .

${ }^{32}$ R. D. Ivany, "Collapse of a cavitation bubble in viscous compressible liquid-Numerical and experimental analysis, Ph.D. thesis, University of Michigan, Ann Arbor, 1965.

${ }^{33}$ P.-W. Yu, "Experimental and numerical examination of cavitating flows," Ph.D. thesis, University of Michigan, Ann Arbor, 1995. 